\title{
Dynamics of branched tissue assembly
}

Sriram Manivannan' and Celeste M Nelson 1,2,*

\begin{abstract}
The assembly of cells into tissues is a complex process controlled by numerous signaling pathways to ensure the fidelity of the final structure. Tissue assembly is also very dynamic, as exemplified by the formation of branched organs. Here we present two examples of tissue assembly in branched systems that highlight this dynamic nature: formation of the tracheal network in Drosophila melanogaster and the ducts of the mammary gland in mice. Extension of the branches during tracheal development is a stereotyped process that produces identical organ geometries across individuals, whereas elongation of the ducts of the pubertal mammary gland is a non-stereotyped process that produces unique patterns. By studying these two organs, we can begin to understand the dynamic nature of development of other stereotyped and nonstereotyped branching systems, including the lung, kidney, and salivary gland.
\end{abstract}

The self assembly of cells into tissues and organs is an elegant and intricate process that is vital for development and homeostasis. During organogenesis, the assembly of cells is controlled genetically as well as through cues from cell-cell and cell-matrix interactions $[1,2]$. Cells assemble into higher-order patterns that are either stereotyped such that there is little variation in the timing and final architecture between individuals, or non-stereotyped, which leads to differences in the details [3]. The branching of the ducts of the pubertal mammary gland is a non-stereotyped process in which the interactions between the ducts and their surrounding microenvironment produce unique tree-like architectures. In contrast, branching of the trachea in Drosophila melanogaster is a stereotyped process that is under strict genetic control. These two systems serve as excellent models with which

\footnotetext{
*Correspondence: celesten@princeton.edu

'Department of Chemical and Biological Engineering, Princeton University, Princeton, NJ 08544, USA

Full list of author information is available at the end of the article
}

to investigate the dynamic interplay between cells during tissue formation, under two different modes of control.

\section{Formation of the trachea in D. melanogaster}

The trachea of the fruit fly is a ductal structure responsible for the delivery of oxygen to tissues. This organ forms during embryonic development and involves invagination, division, extension and fusion of select cells of placodes along the lateral ectoderm (Figure 1). The determination and positioning of the placodes as well as the choreographed steps that lead to the formation of the trachea is predominantly under the control of the gene trachealess $(\operatorname{Trh})[4,5]$. At embryonic stage 11, through the activation of the gene rhomboid (Rho) by Trh, the 20 placodes containing approximately 40 cells each invaginate through apical constriction and undergo mitotic division to form 80-cell tracheal sacs [6-8]. The internalized cells then extend from the sites of invagination to form six distinct branches: the dorsal branch (DB), dorsal trunk (DT), visceral branch (VB), lateral trunk (LT), ganglionic branch (GB), and transverse connective (TC). The TC forms from the mid-region of each sac, and DTs extend along the anterior-posterior axis and fuse with DTs of the neighboring sacs to form the main throughway of the trachea. The cells of the DB migrate dorsally and select fusion cells that bind with their counterpart cells of DBs at the opposite side of the embryo [9], while the cells of the LT migrate ventrally and bind with their counterpart LT cells of neighboring metameres to the anterior and posterior, thus fusing the branches and forming additional contiguous pathways for circulation. The cells of the VB and GB do not fuse with those of the neighboring sacs but still branch and extend into the surroundings to complete the tracheal structure [7]. The extensions of the branches are guided by various signaling pathways, including Breathless (Btl) [10], Decapentaplegic (Dpp) [11] and Slit [12]. Even in a strictly stereotyped branching system such as this, however, the role of cellular dynamics is vital and can be seen in the extension and fusion of the DB.

The DB consists of approximately six cells and these cells migrate dorsally away from the sac toward the morphogen Branchless (Bnl), which acts as a chemoattractant secreted by the surrounding cells [13]. However, the branch is able to extend normally even when all 
(a)

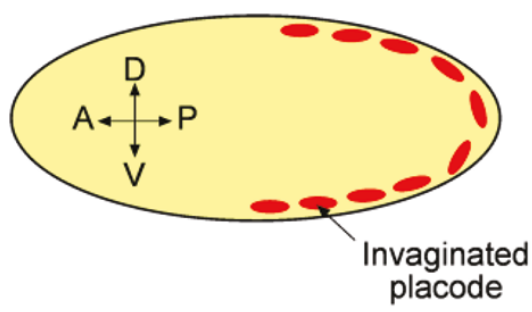

(b)

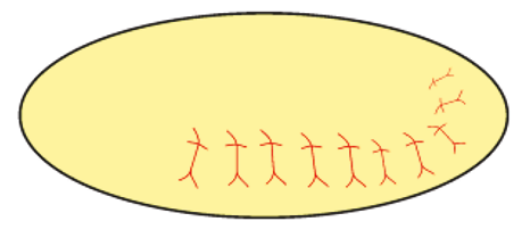

(c)

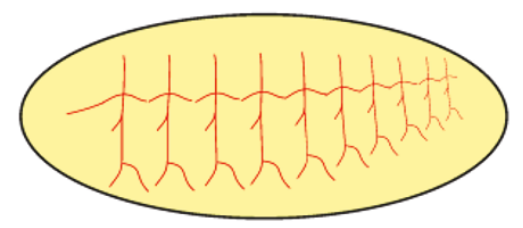

(d)

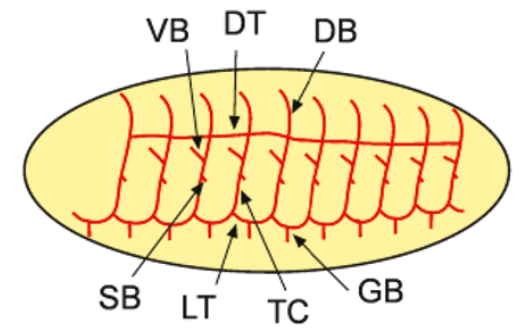

(e)

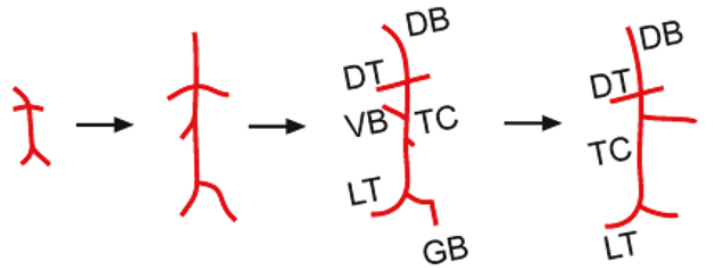

\section{Embryonic Stage $13 \quad$ Stage 16 Pupa Stage 12}

Figure 1. Schematic of tracheal development. (a) At embryonic stage 11 , the placodes have invaginated and are ready to extend stereotypically. (b) At stage 12, the branches begin extension. (c) At stage 13, the branches have fully extended and begin to fuse. (d) By stage 16, the tracheal fusion is complete. Progenitor cells called tracheoblasts that become activated during metamorphosis remodeling are located in the spiracular branch (SB). (e) Schematic of typical tracheal branch development from embryonic stage 12 to stage 13 to stage 16, after the completion of tracheal branching and fusion and to the completion of metamorphosis remodeling. DB, dorsal branch; DT, dorsal trunk; GB, ganglionic branch; LT, lateral trunk; $\mathrm{TC}$, transverse connective; $\mathrm{VB}$, visceral branch. but one of the cells is devoid of Btl, the receptor for Bnl [14]. The cell with functional Btl receptors acts as a leader and is able to guide the rest of the cells. In wild-type embryos, a leader cell emerges even when every cell expresses Btl. At this stage of development, the cells of the DB are nearly identical and there is no predetermined leader; however, there are small differences in the expression level of Btl. By exploiting these small differences, the cells with the highest expression levels of Btl jockey for the lead position in the newly forming branch, while simultaneously amplifying the difference in the activation of Btl among the population through Notch-mediated lateral inhibition [14-17]. The activation of Btl by Bnl induces the production of Delta, a ligand that binds to and activates Notch in the neighboring cells. Activated Notch inhibits signaling downstream of the Btl receptor and production of Delta within that cell. Over time, this results in one cell with substantially higher Btl activity than its neighbors [16,17]. The successful cell assumes the role of the leader at the tip of the branch while the other cells form the stalk. This establishes a hierarchy and the leader cell is able to efficiently move the branch up the Bnl gradient. As the dorsal branch extends, one of the cells of the branch comes in contact with a cell from the neighboring branch and binds to it, thus fusing the branches to form a contiguous airway [9]. Notch signaling again plays a similar role by aiding $\mathrm{Bnl}$ and Dpp and ordaining the fusion cell fate $[16,18,19]$.

During metamorphosis, the trachea once again undergoes significant remodeling. Posterior tracheal branches are lost while anterior branches expand to cover that space and new cells replace most of the cells in the existing branches. Progenitor cells called tracheoblasts that have remained quiescent during embryonic and larval development begin to proliferate and travel along the TC and VB under the guidance of Btl and replace the old cells $[20,21]$. However, the DBs are replaced not by progenitor cells but by differentiated DB cells that reenter the cell cycle and proliferate to replace the existing branch [21].

\section{Formation of the mammary ducts}

The mammary gland is a ductal structure responsible for production and secretion of milk in mammals. The ducts of the mammary gland are composed of a hollow lumen surrounded by a layer of luminal epithelial cells, which are themselves surrounded by a layer of myoepithelial cells that create the basement membrane that separates the epithelium from the stroma. During puberty in rodents, hormones from the ovary and the pituitary gland induce the formation of bulbous terminal end buds (TEBs) at the tips of the rudimentary epithelial ducts [22]. Extension and branching of the TEBs into the surrounding mesenchyme sculpt the highly branched 


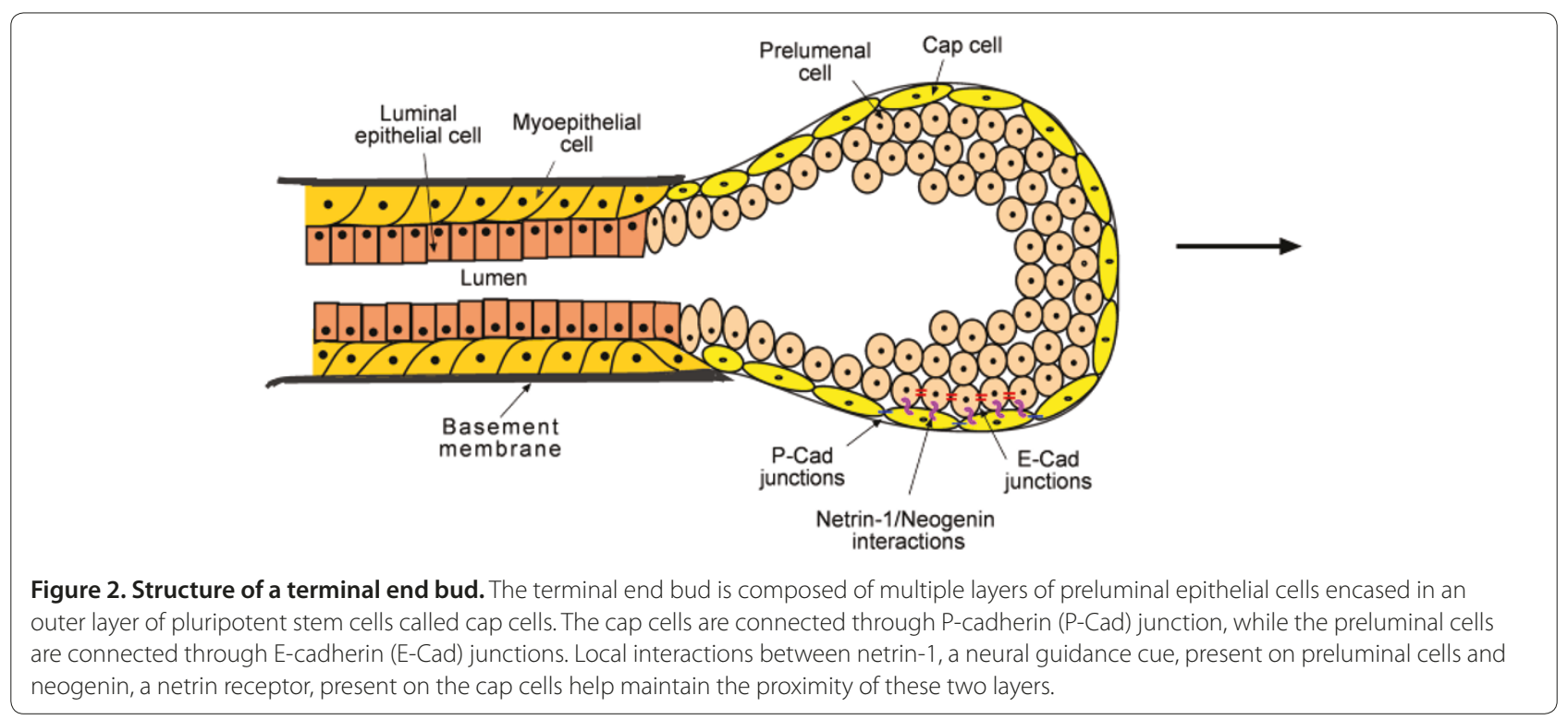

mature mammary epithelial tree. The TEB is composed of multiple layers of preluminal epithelial cells encased in an outer layer of pluripotent stem cells called cap cells (Figure 2) [23]. The cap cells and the preluminal epithelial cells are highly proliferative. The force generated from the rapid proliferation, along with the construction of basement membrane laterally and destruction of extracellular matrix at the front, is thought to propel the ducts forward at an average rate of $500 \mu \mathrm{m}$ per day $[24,25]$. As the TEBs extend, the cap cells move laterally along the duct and differentiate into myoepithelial cells, while the preluminal epithelial cells give rise to luminal epithelial cells [26]. The structure of the TEB is maintained through E-cadherin- and P-cadherin-containing adherens junctions and netrin- $1 /$ neogenin interactions. Preluminal cells interact through E-cadherin, while the cap cells are connected by P-cadherin [27]. Local interactions between netrin-1, a neural guidance cue present on preluminal cells, and neogenin, a netrin receptor present on the cap cells, help maintain the proximity of the preluminal cells to the cap cells [25]. Loss of either netrin or neogenin results in the separation of the cap cell layer from the preluminal layer and destabilization of the TEB [25].

Estrogen and growth hormone initiate ductal elongation by activating the estrogen receptor $(\mathrm{ER} \alpha)$ and the growth hormone receptor, respectively, in the stromal cells surrounding the epithelium [27-29]. The activation of growth hormone receptor enables TEB formation through the insulin-like growth factor pathway [30]. Estrogen signaling leads to the activation of progesterone receptors in the epithelium, which induces epithelial proliferation and ductal movement. Estrogen signaling also stimulates the stromal cells to activate matrix metalloproteinases (MMPs) through amphiregulin and epidermal growth factor receptor [22]. Stromal cells activate several MMPs, including MMP3 and MMP14. MMP14 activates MMP2, which aids in ductal elongation by preventing apoptosis of the cells within the TEB. MMP2 and MMP3 also play a role in determining sites of lateral branching along the duct. High MMP2 prevents the initiation of lateral branches by stimulating the production of transforming growth factor (TGF) $\beta$, whereas MMP3 enables lateral branching by degrading the basement membrane and allowing the cells to extend through the fat pad [31].

Unlike tracheal branching, there is no evidence for a global chemoattractant that guides the TEBs through the fatpad [32]. The TEBs weave through the fatpad, extending lateral branches and bifurcating along the way, by integrating the global stimulatory cues with more local cues from neighboring cells, their environment and their own geometry [32]. Patterns of endogenous mechanical stresses arise along the ducts due to asymmetries in their geometry coupled with isometric contraction of the cells and their ability to transmit these forces to their neighbors through adherens junctions [33]. These mechanical stress patterns appear to regulate the sites at which branches initiate. Indeed, three-dimensional organotypic culture models revealed that branches initiate from sites of highest mechanical stress [32], and that cells in these regions of high mechanical stress adopt a more motile mesenchymal phenotype $[34,35]$, increase signaling through focal adhesion kinase (FAK) [32], and increase production of MMPs to aid in degradation of surrounding matrix, all of which enable branch extension [36]. However, while high mechanical stress is necessary for branch initiation, it is not sufficient [32]. Branch initiation and extension are also controlled by the local 
concentration of morphogens such as TGF $\beta$ [37]. High concentrations of TGF $\beta$ prevent branching of the ducts, and autocrine secretion of this inhibitory morphogen by the epithelial cells is thought to prevent aberrant branching and maintain proper spacing between ducts $[37,38]$. By responding to these various stimulatory and inhibitory cues, the TEBs navigate through the stroma until they reach the limits of the fat pad, where they regress and form terminal ducts. However, the ducts still maintain a certain population of undifferentiated stem cells that are capable of self-renewal and repopulation of the entire mammary gland $[39,40]$. These undifferentiated cells are thought to aid in the remodeling of the mammary gland during and after pregnancy, where further ductal expansion and regression occur, respectively [40].

\section{Conclusion}

Branching of the trachea and the mammary gland highlight the dynamic process of tissue formation. These two systems show how stem cells, progenitor cells and differentiated cells come together, and through a combination of global and local guidance cues specify the final architecture of the tissue. During puberty in mammals, the pluripotent cap cells combined with other cell types of the TEB and through the regulation of the microenvironment produce the unique pattern of the ductal tree. In the formation of $D$. melanogaster trachea, even though the final architecture is fixed, the cells of the invaginated tracheal placodes have a high degree of freedom to determine the leaders, the fusion cells and those that will remain quiescent during the various branch formations. In addition to normal development, the dynamics of stem cells also come into play during tumor formation. Cancer stem cells (CSCs) are specialized cells within tumors that are capable of selfrenewal and initiation of tumors [41]. CSCs have all the characteristics of normal stem cells and are even capable of generating non-malignant structures [42,43]. The origin of CSCs is still hotly debated. While the similarities between CSCs and normal stem cells might strongly suggest that CSCs arise through aberrant differentiation of normal stem cells, there is also evidence to suggest that CSCs might arise from progenitors or even through dedifferentiation of fully differentiated cells. Understanding the dynamics of cells during normal development will help in understanding CSCs and malignant development in general, as many of the processes involved in normal development are conserved in malignant development.

Thanks in large part to advances in technology, we are finally able to glimpse at the complexities of developmental processes. However, better in vivo imaging approaches are needed to fully answer most of the open questions, as it is near impossible to investigate dynamic processes using still images. Advances in live imaging could help, for example, to study the formation and movement of the TEBs. Studies in culture have suggested that organization of the TEBs might involve cellular sorting to optimize the structure for invasion through the fatpad [44]; however, this has yet to be shown in vivo. Furthermore, the mechanism by which the TEB moves through the fatpad is still unclear [45]. In addition to imaging, advances in techniques to manipulate in vivo microenvironments might prove to be the most fruitful venture. The mechanics of the microenvironment integrate with molecular cues in the regulation of cell division, motility, branching, and stem cell differentiation [46]. Laser ablation studies in D. melagonaster have been used to show the involvement of tensile forces in tracheal branching [47-49]. At present, however, culture models still provide one of the best means of studying the effects of the mechanics of the microenvironment on these processes in a controlled and quantifiable manner. While they do suffer from drawbacks of artificiality, sophisticated three-dimensional culture models can aid to bridge the gap between the plastic world and the in vivo world, and could play an important role in studying the interplay between multiple cell types during development $[50,51]$. A better appreciation of the dynamic nature of tissue assembly will have a great impact on the study of normal and malignant development and may also will help advance the field of tissue engineering.

\section{This article is part of a thematic series on Physical influences on stem cells edited by Gordana Vunjak-Novakovic. Other articles in the series can be found online at http://stemcellres.com/series/physical}

\section{Abbreviations}

BNL, Branchless; BTL, Breathless; CSC, cancer stem cell; DB, dorsal branch; DPP, Decapentaplegic; DT, dorsal trunk; GB, ganglionic branch; LT, lateral trunk; MMP, matrix metalloproteinase; TC, transverse connective; TEB, terminal end bud; TGF, transforming growth factor; Trh, Trachealess; VB, visceral branch.

\section{Competing interests}

The authors declare that they have no competing interests.

\section{Acknowledgements}

Work from the authors' lab was supported in part by the NIH (CA128660, GM083997, and HL110335), Susan G Komen for the Cure, the David and Lucile Packard Foundation, and the Alfred P Sloan Foundation. CMN holds a Career Award at the Scientific Interface from the Burroughs Wellcome Fund.

\section{Author details}

'Department of Chemical and Biological Engineering, Princeton University, Princeton, NJ 08544, USA. ²Department of Molecular Biology, Princeton University, Princeton, NJ 08544, USA.

Published: 31 October 2012

\section{References}

1. Davies JA: Self-organization as a tool in mammalian tissue engineering. In Advances in Regenerative Medicine. Edited by Wislet-Gendebien S. In Tech; 2011:261-274.

2. Whitesides GM, Grzybowski B: Self-assembly at all scales. Science 2002, 295:2418-2421. 
3. Gjorevski N, Nelson CM: Integrated morphodynamic signalling of the mammary gland. Nat Rev Mol Cell Bio 2011, 12:581-593.

4. Chung S, Chavez C, Andrew DJ: Trachealess (Trh) regulates all tracheal genes during Drosophila embryogenesis. Dev Biol 2011, 360:160-172.

5. Nishimura $M$, Inoue $Y$, Hayashi $S$ : A wave of EGFR signaling determines cell alignment and intercalation in the Drosophila tracheal placode. Development 2007, 134:4273-4282.

6. Kerman BE, Cheshire AM, Andrew DJ: From fate to function: the Drosophila trachea and salivary gland as models for tubulogenesis. Differentiation 2006, 74:326-348.

7. Maruyama R, Andrew DJ: Drosophila as a model for epithelial tube formation. DevDynam 2012, 241:119-135.

8. Petit V, Ribeiro C, Ebner A, Affolter M: Regulation of cell migration during tracheal development in Drosophila melanogaster. Int J Dev Biol 2002, 46:125-132.

9. Gervais $L$, Lebreton G, Casanova J: The making of a fusion branch in the Drosophila trachea. Dev Biol 2012, 362:187-193.

10. Klambt C, Glazer L, Shilo BZ: breathless, A Drosophila FGF receptor homolog, is essential for migration of tracheal and specific midline glial cells. Genes Dev 1992, 6:1668-1678.

11. Vincent S, Ruberte E, Grieder NC, Chen CK, Haerry T, Schuh R, Affolter M: DPP controls tracheal cell migration along the dorsoventral body axis of the Drosophila embryo. Development 1997, 124:2741-2750.

12. Englund C, Steneberg P, Falileeva L, Xylourgidis N, Samakovlis C: Attractive and repulsive functions of Slit are mediated by different receptors in the Drosophila trachea. Development 2002, 129:4941-4951.

13. Metzger RJ, Krasnow MA: Genetic control of branching morphogenesis. Science 1999, 284:1635-1639.

14. Ghabrial AS, Krasnow MA: Social interactions among epithelial cells during tracheal branching morphogenesis. Nature 2006, 441:746-749.

15. Hacohen N, Kramer S, Sutherland D, Hiromi Y, Krasnow MA: sprouty encodes a novel antagonist of FGF signaling that patterns apical branching of the Drosophila airways. Cell 1998, 92:253-263.

16. Ikeya T, Hayashi S: Interplay of Notch and FGF signaling restricts cell fate and MAPK activation in the Drosophila trachea. Development 1999 126:4455-4463.

17. Ohshiro T, Emori Y, Saigo K: Ligand-dependent activation of breathless FGF receptor gene in Drosophila developing trachea. Mech Dev 2002, 114:3-11.

18. Steneberg P, Hemphälä J, Samakovlis C: Dpp and Notch specify the fusion cell fate in the dorsal branches of the Drosophila trachea. Mech Dev 1999 87:153-163

19. Samakovlis C, Manning G, Steneberg P Hacohen N, Cantera R, Krasnow MA: Genetic control of epithelial tube fusion during Drosophila tracheal development. Development 1996, 122:3531-3536

20. Sato M, Kornberg TB: FGF is an essential mitogen and chemoattractant for the air sacs of the Drosophila tracheal system. Dev Cell 2002, 3:195-207.

21. Weaver M, Krasnow MA: Dual origin of tissue-specific progenitor cells in Drosophila tracheal remodeling. Science 2008, 321:1496-1499.

22. Sternlicht M: Key stages in mammary gland development: The cues that regulate ductal branching morphogenesis. Breast Cancer Res 2006, 8:201.

23. Hinck L, Silberstein G: Key stages in mammary gland development: The mammary end bud as a motile organ. Breast Cancer Res 2005, 7:245-251.

24. Silberstein $\mathrm{G}$ : Role of the stroma in mammary development. Breast Cancer Res 2001, 3:218-224.

25. Srinivasan K, Strickland P, Valdes A, Shin G, Hinck L: Netrin-1/neogenin interaction stabilizes multipotent progenitor cap cells during mammary gland morphogenesis. Dev Cell 2003, 4:371-382.

26. Richert MM, Schwertfeger KL, Ryder JW, Anderson SM: An atlas of mouse mammary gland development. J Mammary Gland Biol 2000, 5:227-241.

27. Silberstein G: Postnatal mammary gland morphogenesis. Microsc Res Techniq 2001, 52:155-162

28. Wiseman BS, Werb Z: Stromal effects on mammary gland development and breast cancer. Science 2002, 296:1046-1049.

29. Nelson CM, Bissell MJ: Of extracellular matrix, scaffolds, and signaling: tissue architecture regulates development, homeostasis, and cancer. Annu Rev Cell Dev Biol 2006, 22:287.

30. Ruan W, Kleinberg DL: Insulin-like growth factor I is essential for terminal end bud formation and ductal morphogenesis during mammary development. Endocrinology 1999, 140:5075-5081.
31. Wiseman BS, Sternlicht MD, Lund LR, Alexander CM, Mott J, Bissell MJ, Soloway P, Itohara S, Werb Z: Site-specific inductive and inhibitory activities of MMP- 2 and MMP-3 orchestrate mammary gland branching morphogenesis. J Cell Biol 2003, 162:1 123-1133.

32. Gjorevski N, Nelson CM: Endogenous patterns of mechanical stress are required for branching morphogenesis. Integr Biol 2010, 2:424-434.

33. Nelson CM, Jean RP, Tan JL, Liu WF, Sniadecki NJ, Spector AA, Chen CS: Emergent patterns of growth controlled by multicellular form and mechanics. Proc Natl Acad SciU S A 2005, 102:11594-11599.

34. Gomez EW, Chen QK, Gjorevski N, Nelson CM: Tissue geometry patterns epithelial-mesenchymal transition via intercellular mechanotransduction. J Cell Biochem 2010, 110:44-51.

35. Lee K, Gjorevski N, Boghaert E, Radisky DC, Nelson CM: Snail1, Snail2, and E47 promote mammary epithelial branching morphogenesis. EMBO $\rfloor$ 2011, 30:2662-2674.

36. Moore KA, Polte T, Huang S, Shi B, Alsberg E, Sunday ME, Ingber DE: Control of basement membrane remodeling and epithelial branching morphogenesis in embryonic lung by Rho and cytoskeletal tension. Dev Dynam 2005, 232:268-281

37. Nelson CM, VanDuijn MM, Inman JL, Fletcher DA, Bissell MJ: Tissue geometry determines sites of mammary branching morphogenesis in organotypic cultures. Science 2006, 314:298-300

38. Pavlovich AL, Boghaert E, Nelson CM: Mammary branch initiation and extension are inhibited by separate pathways downstream of TGF $\beta$ in culture. Exp Cell Res 2011, 317:1872-1884

39. Stingl J, Eirew P, Ricketson I, Shackleton M, Vaillant F, Choi D, Li HI, Eaves CJ: Purification and unique properties of mammary epithelial stem cells. Nature 2006, 439:993-997.

40. Shackleton M, Vaillant F, Simpson KJ, Stingl J, Smyth GK, Asselin-Labat M-L, Wu L, Lindeman GJ, Visvader JE: Generation of a functional mammary gland from a single stem cell. Nature 2006, 439:84-88

41. Zucchi I, Sanzone S, Astigiano S, Pelucchi P, Scotti M, Valsecchi V, Barbieri O, Bertoli G, Albertini A, Reinbold RA, Dulbecco R: The properties of a mammary gland cancer stem cell. Proc Natl Acad Sci U S A 2007, 104:10476-10481.

42. Kordon EC, Smith GH: An entire functional mammary gland may comprise the progeny from a single cell. Development 1998, 125:1921-1930.

43. Charafe-Jauffret E, Ginestier C, lovino F, Wicinski J, Cervera N, Finetti P, Hur M-H, Diebel ME, Monville F, Dutcher J, Brown M, Viens P, Xerri L, Bertucci F, Stassi G, Dontu G, Birnbaum D, Wicha MS: Breast cancer cell lines contain functional cancer stem cells with metastatic capacity and a distinct molecular signature. Cancer Res 2009, 69:1302-1313.

44. Mori H, Gjorevski N, Inman JL, Bissell MJ, Nelson CM: Self-organization of engineered epithelial tubules by differential cellular motility. Proc Natl Acad Sci U S A 2009, 106:14890-14895.

45. Silberstein G, Daniel C: Glycosaminoglycans in the basal lamina and extracellular matrix of the developing mouse mammary duct. Dev Biol 1982, 90:215-222

46. Lui C, Lee K, Nelson C: Matrix compliance and RhoA direct the differentiation of mammary progenitor cells. Biomech Model Mechanobiol 2011. [Epub ahead of print]

47. Caussinus E, Colombelli J, Affolter M: Tip-cell migration controls stalk-cell intercalation during Drosophila tracheal tube elongation. Curr Bio/ 2008 , 18:1727-1734

48. Shaye DD, Casanova J, Llimargas M: Modulation of intracellular trafficking regulates cell intercalation in the Drosophila trachea. Nat Cell Biol 2008, 10:964-970.

49. Baum B, Georgiou M: Dynamics of adherens junctions in epithelial establishment, maintenance, and remodeling. J Cell Biol 2011, 192:907-917.

50. Pavlovich AL, Manivannan S, Nelson CM: Adipose stroma induces branching morphogenesis of engineered epithelial tubules. Tissue Eng Pt A 2010, 16:3719-3726.

51. Bhowmick NA, Neilson EG, Moses HL: Stromal fibroblasts in cancer initiation and progression. Nature 2004, 432:332-337.

doi:10.1186/scrt133

Cite this article as: Manivannan S, Nelson CM: Dynamics of branched tissue assembly. Stem Cell Research \& Therapy 2012, 3:42. 